\title{
A SIMPLE PROOF OF INEQUALITIES RELATED TO MEANS
}

\author{
GOU-SHENG YANG AND SHUOH-JUNG LIU
}

\begin{abstract}
The purpose of this paper is to give a fairly elementary method to prove that the function $u(\alpha)=\left[\frac{x^{\alpha}-y^{\alpha}}{\alpha(x-y)}\right]^{\frac{1}{\alpha-1}}, \alpha \neq 0,1, u(0)=I, u(1)=L$, is strictly increasing, and to give a simple proof of the inequalities $x<H<\frac{x y}{I}<\frac{x y}{L}<G<$ $L<I<A<y$, where $0<x<y$ and $A, G, H, I, L$ are the arithmetic, the geometric, the harmonic, the identric and logarithmic means of $x$ and $y$, respectively.
\end{abstract}

\section{Introduction}

Given two positive real numbers $x$ and $y$, the arithmetic mean $A(x, y)$, the geometric mean $G(x, y)$, the logarithmic mean $L(x, y)$, the identric mean $I(x, y)$ and the harmonic mean $H(x, y)$ of $x$ and $y$ are defined, respectively, by

$$
\begin{gathered}
A=A(x, y)=\frac{x+y}{2}, \quad G=G(x, y)=\sqrt{x y}, \quad H=H(x, y)=\frac{2 x y}{x+y}, \\
L=L(x, y)=\left\{\begin{array}{cc}
\frac{x-y}{\ln x-\ln y}, & x \neq y, \quad I=I(x, y)=\left\{\begin{array}{c}
e^{-1}\left(\frac{x^{x}}{y^{y}}\right)^{\frac{1}{x-y}}, x \neq y, \\
x \quad, x=y .
\end{array} \quad, x=y .\right.
\end{array}\right.
\end{gathered}
$$

It is known that (see[1], p.130), if $0<x<y$, then

$$
x<H<\frac{x y}{I}<\frac{x y}{L}<G<L<I<A<y .
$$

Throughout, we assume $0<x<y$.

In [5], K. B. Stolarsky, defined the function

$$
u(\alpha)=\left\{\begin{aligned}
{\left[\frac{x^{\alpha}-y^{\alpha}}{\alpha(x-y)}\right]^{\frac{1}{\alpha-1}}, } & \alpha \neq 0,1, \\
L(x, y) & , \alpha=0, \\
I(x, y) & , \alpha=1 .
\end{aligned}\right.
$$

Corresponding author: Gou-Sheng Yang.

2000 Mathematics Subject Classification. 26D15.

Key words and phrases. Arithmetic mean, geometric mean, harmonic mean, logarithmic mean, identric mean. 
and proved that $u(\alpha)$ satisfies the following properties:

(i) $0<x<u(\alpha)<y, \forall \alpha \in \mathbb{R}$.

(ii) $u(\alpha)$ is strictly increasing in $\alpha$, and $u(\alpha)$ approaches to $y$ (or $x$ ) as $\alpha$ approaches to $\infty($ or $-\infty)$.

(iii) $u(\alpha)$ is continuous in $\alpha$, and $u(-1)=G(x, y), \quad u(0)=L(x, y), \quad u(1)=I(x, y), \quad u(2)=A(x, y)$.

The purpose of this paper is to give a fairly elementary method to prove that the function $u(\alpha)$ is strictly increasing and to give a simple proof of the inequalities (1).

\section{A simple proof of the monotone of $u(\alpha)$.}

To prove that $u(\alpha)$ is strictly increasing, we need the following Lemmas:

Lemma 1. Let $g(t)=t(\ln t)^{2}-(t-1)^{2}, t>0$. Then $g(t)<0, \forall t \in(0,1) \cup(1, \infty)$.

Proof. For $t>0$, we have

$$
\begin{aligned}
g^{\prime}(t) & =2 \ln t+(\ln t)^{2}-2(t-1), \\
g^{\prime \prime}(t) & =\frac{2}{t}+\frac{2 \ln t}{t}-2, \\
g^{\prime \prime \prime}(t) & =\frac{-2 \ln t}{t^{2}},
\end{aligned}
$$

it follows that, if $t>1$, then $g^{\prime \prime \prime}(t)<0$, so that $g^{\prime \prime}(t)<g^{\prime \prime}(1)=0$, which implies that $g^{\prime}(t)$ is strictly decreasing on $(1, \infty)$, and hence $g^{\prime}(t)<g^{\prime}(1)=0$, which, again, implies that $\mathrm{g}(\mathrm{t})$ is strictly decreasing on $(1, \infty)$. Therefore $g(t)<g(1)=0$.

Next, if $0<t<1$, then $g^{\prime \prime \prime}(t)>0$, so that $g^{\prime \prime}(t)<g^{\prime \prime}(1)=0$, which implies that $g^{\prime}(t)$ is strictly decreasing on $(0,1)$, and then $g^{\prime}(t)>g^{\prime}(1)=0$.

Hence $g(t)$ is strictly increasing on $(0,1)$. Therefore $g(t)<g(1)=0$.

This completes the proof.

Lemma 2. If $b>1$, let

$$
f(\alpha)=\left\{\begin{array}{cc}
\frac{(\alpha-1) b^{\alpha} \ln b}{b^{\alpha}-1}-1+\frac{1}{\alpha}-\ln \frac{b^{\alpha}-1}{\alpha(b-1)}, & \alpha \neq 0, \\
\ln \frac{b-1}{\ln b}-\ln \sqrt{b} & , \quad \alpha=0 .
\end{array}\right.
$$

Then $f(\alpha)>0, \forall \alpha \neq 1$. 
Proof. For $\alpha \neq 0$, we have

$$
\begin{aligned}
f^{\prime}(\alpha) & =\frac{\left(b^{\alpha}-1\right) b^{\alpha} \ln b[(\alpha-1) \ln b+1]-(\alpha-1) b^{2 \alpha}(\ln b)^{2}}{\left(b^{\alpha}-1\right)^{2}}-\frac{1}{\alpha^{2}}-\frac{\alpha b^{\alpha} \ln b-b^{\alpha}+1}{\alpha\left(b^{\alpha}-1\right)} \\
& =\frac{1-\alpha}{\alpha^{2}\left(b^{\alpha}-1\right)^{2}}\left[b^{\alpha}\left(\ln b^{\alpha}\right)^{2}-\left(b^{\alpha}-1\right)^{2}\right] \\
& =\frac{1-\alpha}{\alpha^{2}\left(b^{\alpha}-1\right)^{2}} g\left(b^{\alpha}\right) .
\end{aligned}
$$

where the function $g$ is defined as in Lemma 1 ..

If $\alpha>1$, it follows from (2) and Lemma 1. that $f^{\prime}(\alpha)>0$, so that $f(\alpha)$ is strictly increasing on $(1, \infty)$. Hence $f(\alpha)>f(1)=0$.

If $0<\alpha<1$, it follows from (2) and Lemma 1. that $f^{\prime}(\alpha)<0$, so that $f(\alpha)$ is strictly decreasing on $(0,1)$. Hence $f(\alpha)>f(1)=0$.

If $\alpha<0$, it follows from (2) and Lemma 1. that $f^{\prime}(\alpha)<0$, so that $f(\alpha)>f(0)>0$.

To show $f(0)>0$, we consider the function $F(x)=\sqrt{x}-\frac{1}{\sqrt{x}}-\ln x, \quad x>1$. Differentiating gives $F^{\prime}(x)=\frac{(\sqrt{x}-1)^{2}}{2 x \sqrt{x}}>0$, so that $F(x)>F(1)=0$. This implies that $f(0)>0$. Consequently, $f(\alpha)>0, \forall \alpha \neq 1$.

Now we are ready to prove that the function $u(\alpha)$ is strictly increasing in $\alpha$.

Proof. Let $b=\frac{y}{x}>1$, and $v(\alpha)=\frac{u(\alpha)}{x}$. Then

$$
v(\alpha)=\left\{\begin{array}{cc}
{\left[\frac{b^{\alpha}-1}{\alpha(b-1)}\right]^{\frac{1}{\alpha-1}}, \quad \alpha \neq 0,1,} \\
\frac{L(x, y)}{x} \quad, \quad \alpha=0, \\
\frac{I(x, y)}{x} \quad, \quad \alpha=1 .
\end{array}\right.
$$

It suffices to show that $v(\alpha)$ is strictly increasing in $\alpha$.

Now, if $\alpha \neq 0,1$, we have

$$
\begin{aligned}
v^{\prime}(\alpha) & =v(\alpha) \frac{(\alpha-1)\left[\frac{\alpha(b-1)}{b^{\alpha}-1} \frac{\alpha b^{\alpha} \ln b-\left(b^{\alpha}-1\right)}{\alpha^{2}(b-1)}\right]-\ln \frac{b^{\alpha}-1}{\alpha(b-1)}}{(\alpha-1)^{2}} \\
& =\frac{v(\alpha)}{(\alpha-1)^{2}}\left[\frac{(\alpha-1)\left(\alpha b^{\alpha} \ln b-b^{\alpha}+1\right)}{\alpha\left(b^{\alpha}-1\right)}-\ln \frac{b^{\alpha}-1}{\alpha(b-1)}\right] \\
& =\frac{v(\alpha)}{(\alpha-1)^{2}}\left[\frac{(\alpha-1) b^{\alpha} \ln b}{b^{\alpha}-1}-1+\frac{1}{\alpha}-\ln \frac{b^{\alpha}-1}{\alpha(b-1)}\right] \\
& =\frac{v(\alpha)}{(\alpha-1)^{2}} f(\alpha),
\end{aligned}
$$


where $f(\alpha)$ is defined as in Lemma 2 ..

Since $v(\alpha)>0$, it follows from (3) and Lemma 2. that $v^{\prime}(\alpha)>0$, so that $v(\alpha)$ is strictly increasing for $\alpha \neq 0,1$.

Observe that

$$
\begin{aligned}
v^{\prime}(0) & =\lim _{\alpha \rightarrow 0} \frac{v(\alpha)-v(0)}{\alpha-0} \\
& =\lim _{\alpha \rightarrow 0} \frac{v(\alpha)}{(\alpha-1)^{2}} f(\alpha) \\
& =\frac{L(x, y)}{y} \lim _{\alpha \rightarrow 0} f(\alpha) \\
& =\frac{L(x, y)}{y}\left(\ln \frac{b-1}{\ln b}-\ln \sqrt{b}\right)>0 . \\
v^{\prime}(1) & =\lim _{\alpha \rightarrow 1} \frac{v(\alpha)-v(1)}{\alpha-1} \\
& =\lim _{\alpha \rightarrow 1} \frac{v(\alpha)}{(\alpha-1)^{2}} f(\alpha) \\
& =\left[\lim _{\alpha \rightarrow 1} v(\alpha)\right]\left[\lim _{\alpha \rightarrow 1} \frac{f^{\prime}(\alpha)}{2(\alpha-1)}\right] \\
& =\frac{I(x, y)}{y}\left[\lim _{\alpha \rightarrow 1} \frac{1}{2(\alpha-1)} \frac{1-\alpha}{\alpha^{2}\left(b^{\alpha}-1\right)^{2}} g\left(b^{\alpha}\right)\right] \\
& =\frac{I(x, y) g(b)}{-2 y(b-1)^{2}}>0 .
\end{aligned}
$$

Consequently, $v^{\prime}(\alpha)>0$ for all $\alpha$.

\section{A simple proof of the inequalities (1)}

Let $w(\alpha)=\frac{x y}{u(\alpha)}$. Then

$$
w(\alpha)=\left\{\begin{array}{cc}
x y\left[\frac{\alpha(x-y)}{x^{\alpha}-y^{\alpha}}\right]^{\frac{1}{\alpha-1}}, & \alpha \neq 0,1, \\
\frac{x y}{L(x, y)} & \alpha=0, \\
\frac{x y}{I(x, y)} & , \quad \alpha=1 .
\end{array}\right.
$$

Since $u(\alpha)$ is strictly increasing in $\alpha$, so that $w(\alpha)$ is strictly decreasing in $\alpha$.

$\lim _{\alpha \rightarrow \infty} w(\alpha)=x, \quad w(2)=H(x, y), \quad w(1)=\frac{x y}{I(x, y)}, \quad w(0)=\frac{x y}{L(x, y)}$, 


$$
w(-1)=G(x, y), \quad w(-2)=\left(A G^{2}\right)^{\frac{1}{3}}, \quad \lim _{\alpha \rightarrow-\infty} w(\alpha)=y .
$$

We have

$$
x<H(x, y)<\frac{x y}{I(x, y)}<\frac{x y}{L(x, y)}<G(x, y)<\left(A G^{2}\right)^{\frac{1}{3}}<y
$$

To show that

$$
\left(A G^{2}\right)^{\frac{1}{3}}<L(x, y)<I(x, y)<A(x, y)
$$

we consider the function $h(\alpha)=A^{\alpha} G^{1-\alpha}, \alpha \in[0,1]$, we see that $h^{\prime}(\alpha)=A^{\alpha} G^{1-\alpha} \ln A-$ $A^{\alpha} G^{1-\alpha} \ln G>0$. Hence $h(\alpha)$ is strictly increasing in $\alpha$.

For $c=\sqrt{\frac{y}{x}}>1$, let

$$
\alpha_{1}=\frac{\ln \frac{c^{2}-1}{2 c \ln c}}{\ln \frac{c^{2}+1}{2 c}}, \quad \alpha_{2}=\frac{-1+\frac{c^{2}+1}{c^{2}-1} \ln c}{\ln \frac{c^{2}+1}{2 c}} .
$$

Then $h\left(\alpha_{1}\right)=L(x, y)$ and $h\left(\alpha_{2}\right)=I(x, y)$.

Observe that $h\left(\frac{1}{3}\right)=\left(A G^{2}\right)^{\frac{1}{3}}$ and $h(1)=A(x, y)$.

To complete the proof of the inequalities (5), it suffices to prove that $\frac{1}{3}<\alpha_{1}<\alpha_{2}<1$.

In order to prove that $\alpha_{1}>\frac{1}{3}$, we need the following Lemma:

Lemma 3. Let $k(x)=2\left(x^{4}+4 x^{2}+1\right) \ln x-3\left(x^{4}-1\right), x>0$. Then $k(x)>0, \forall x>1$.

Proof. Let

$$
\begin{aligned}
& p(x)=\left(4 x^{4}+8 x^{2}\right) \ln x-5 x^{4}+4 x^{2}+1, \\
& q(x)=\left(x^{2}+1\right) \ln x-\left(x^{2}-1\right), \\
& r(x)=2 x^{2} \ln x-x^{2}+1 .
\end{aligned}
$$

Then

$$
\begin{aligned}
k^{\prime}(x) & =2\left(4 x^{3}+8 x\right) \ln x+\frac{-10 x^{4}+8 x^{2}+2}{x} \\
& =\frac{2}{x} p(x) \\
p^{\prime}(x) & =16 x\left(x^{2}+1\right) \ln x-16 x\left(x^{2}-1\right) \\
& =(16 x) q(x), \\
q^{\prime}(x) & =2 x \ln x-x+\frac{1}{x} \\
& =\frac{1}{x} r(x), \\
r^{\prime}(x) & =4 x \ln x .
\end{aligned}
$$

If $x>1$, then $r^{\prime}(x)>0$, so that $r(x)>r(1)=0$, which implies that $q(x)$ is strictly increasing on $(1, \infty)$, then $q(x)>q(1)=0$, which implies that $p(x)$ is strictly increasing on $(1, \infty)$, then $p(x)>p(1)=0$, which again, implies that $k(x)$ is strictly increasing on $(1, \infty)$. 
Consequently, $k(x)>k(1)=0, \forall x>1$.

This completes the proof of the Lemma.

To prove that $\alpha_{1}>\frac{1}{3}$, let

$$
s(x)=\left\{\begin{array}{cc}
\ln \frac{x^{2}-1}{2 x \ln x}-\frac{\ln \frac{x^{2}+1}{2 x}}{3}, & x>1, \\
0 \quad, & x=1 .
\end{array}\right.
$$

Then for $x>1$, we have

$$
\begin{aligned}
s^{\prime}(x) & =\frac{2 x \ln x}{x^{2}-1} \frac{2 x(2 x \ln x)-\left(x^{2}-1\right)(2+2 \ln x)}{4 x^{2}(\ln x)^{2}}-\frac{2 x}{3\left(x^{2}+1\right)} \frac{4 x^{2}-2\left(x^{2}+1\right)}{4 x^{2}} \\
& =\frac{x^{2} \ln x-x^{2}+1+\ln x}{x\left(x^{2}-1\right) \ln x}-\frac{x^{2}-1}{3 x\left(x^{2}+1\right)} \\
& =\frac{k(x)}{3 x\left(x^{4}-1\right) \ln x},
\end{aligned}
$$

where $k(x)$ is defined as in Lemma 3 .

It follows from (6) and Lemma 3. that $s^{\prime}(x)>0, \forall x>1$. Hence $s(x)>s(1)=$ $0, \forall x>1$, which is equivalent to $\frac{1}{3}<\alpha_{1}$. Therefore,

$$
h\left(\frac{1}{3}\right)=\left(A G^{2}\right)^{\frac{1}{3}}<h\left(\alpha_{1}\right)=L(x, y)
$$

(see [2], [3], [4]).

In order to prove that $\alpha_{2}>\alpha_{1}$, we need the following Lemma:

Lemma 4. Let $\ell(x)=\left(x^{2}-1\right)^{2}-(2 x \ln x)^{2}, x>1$. Then $\ell(x)>0, \forall x>1$.

Proof. Let $\ell_{1}(x)=x^{2}-1-2 x \ln x, x>1$.

It suffices to show $\ell_{1}(x)>0, \forall x>1$. Differentiating gives

$$
\begin{aligned}
& \ell_{1}^{\prime}(x)=2 x-2-2 \ln x, \\
& \ell_{1}^{\prime \prime}(x)=2-\frac{2}{x} .
\end{aligned}
$$

If $x>1$, then $\ell_{1}^{\prime \prime}(x)>0$, so that $\ell_{1}^{\prime}(x)$ is strictly increasing on $(1, \infty)$, and then $\ell_{1}^{\prime}(x)>$ $\ell_{1}^{\prime}(1)=0$, which, implies that $\ell_{1}(x)$ is strictly increasing on $(1, \infty)$.

Consequently, $\ell_{1}(x)>\ell_{1}(1)=0, \forall x>1$.

This completes the proof of the lemma.

To prove that $\alpha_{2}=\frac{-1+\frac{c^{2}+1}{c^{2}-1} \ln c}{\ln \frac{c^{2}+1}{2 c}}>\frac{\ln \frac{c^{2}-1}{2 c \ln c}}{\ln \frac{c^{2}+1}{2 c}}=\alpha_{1}$, let

$$
\ell_{2}(x)=\left\{\begin{array}{cc}
-1+\frac{x^{2}+1}{x^{2}-1} \ln x-\ln \frac{x^{2}-1}{2 x \ln x}, & x>1 \\
0 & , \quad x=1 .
\end{array}\right.
$$


It suffices to show that : $\ell_{2}(x)>0, \forall x>1$.

To this end, we observe that for $x>1$, we have

$$
\begin{aligned}
\ell_{2}^{\prime}(x) & =\frac{2 x\left(x^{2}-1\right)-2 x\left(x^{2}+1\right)}{\left(x^{2}-1\right)^{2}} \ln x+\frac{x^{2}+1}{x\left(x^{2}-1\right)}-\frac{2 x \ln x}{x^{2}-1} \frac{4 x^{2} \ln x-\left(x^{2}-1\right)(2+2 \ln x)}{4 x^{2}(\ln x)^{2}} \\
& =\frac{-4 x^{2}(\ln x)^{2}+\left(x^{4}-1\right) \ln x+\left(1-x^{2}\right)\left(x^{2} \ln x-x^{2}+\ln x+1\right)}{x\left(x^{2}-1\right)^{2} \ln x} \\
& =\frac{\ell(x)}{x\left(x^{2}-1\right)^{2} \ln x}
\end{aligned}
$$

where $\ell(x)$ is defined as in Lemma 4 ..

It follows from (8) and Lemma 4. that $\ell_{2}^{\prime}(x)>0, \forall x>1$, so that $\ell_{2}(x)$ is strictly increasing on $(1, \infty)$. Hence $\ell_{2}(x)>\ell_{2}(1)=0$. Therefore

$$
h\left(\alpha_{2}\right)=I(x, y)>h\left(\alpha_{1}\right)=L(x, y) .
$$

To prove that $\alpha_{2}<1$, we need the following Lemma:

Lemma 5. Let $m(x)=x^{4} \ln x-x^{4}+x^{2} \ln x+x^{2}, x>0$. Then $m(x)>0, \forall x>1$.

Proof. We have

$$
\begin{aligned}
m^{\prime}(x) & =4 x^{3} \ln x-3 x^{3}+2 x \ln x+3 x \\
m^{\prime \prime}(x) & =12 x^{2} \ln x-5 x^{2}+2 \ln x+5 \\
m^{\prime \prime \prime}(x) & =24 x \ln x+2 x+\frac{2}{x} .
\end{aligned}
$$

If $x>1$, then $m^{\prime \prime \prime}(x)>0$, so that $m^{\prime \prime}(x)>m^{\prime \prime}(1)=0$, implies $m^{\prime}(x)$ is strictly increasing on $(1, \infty)$, and $m^{\prime}(x)>m^{\prime}(1)=0$, which again, implies that $m(x)$ is strictly increasing on $(1, \infty)$.

Consequently, $m(x)>m(1)=0, \forall x>1$.

This completes the proof of the lemma.

Now, let

$$
n(x)=\left\{\begin{array}{cc}
\ln \frac{x^{2}+1}{2 x}+1-\frac{x^{2}+1}{x^{2}-1} \ln x, & x>1, \\
0 & , \quad x=1 .
\end{array}\right.
$$

Then for $x>1$, we have

$$
\begin{aligned}
n^{\prime}(x) & =\frac{2 x}{x^{2}+1} \frac{4 x^{2}-2 x^{2}-2}{4 x^{2}}-\frac{\left(x^{2}-1\right)\left(2 x \ln x+x+\frac{1}{x}\right)-2 x\left(x^{2} \ln x+\ln x\right)}{\left(x^{2}-1\right)^{2}} \\
& =\frac{x^{2}-1}{x\left(x^{2}+1\right)}-\frac{x^{3}-4 x \ln x-\frac{1}{x}}{\left(x^{2}-1\right)^{2}} \\
& =\frac{4 m(x)}{x\left(x^{2}+1\right)\left(x^{2}-1\right)^{2}},
\end{aligned}
$$


where $m(x)$ is defined as in Lemma $5 .$.

It follows from 10 and Lemma 5. that $n^{\prime}(x)>0, \forall x>1$.

Hence $n(x)>n(1)=0, \forall x>1$, which is equivalent to $\alpha_{2}<1$. Therefore,

$$
h\left(\alpha_{2}\right)=I(x, y)<h(1)=A(x, y) .
$$

The inequalities (1) then follows from (4), (7) (9) and (11).

\section{References}

[1] P. S. Bullen, D. S. Mitrinović and P. M. Vasić, Means and Their Inequalities, D. Reidle Publishing Company, Dordrecht, Holland, 1988.

[2] B. C. Carlson, The logarithmic mean, Amer. Math. Monthly 79 (1972), 615-618.

[3] E. B. Leach and M. C. Sholander, Extend mean values, ii, J. Math. Anal. 92 (1983), 207223.

[4] J. Sandor, A note on some inequalities for means, Arch. Math. (Basel) 56 (1991), 471-473.

[5] K. B. Stolarsky, Generalizations of the logarithmic mean, Mathematics Magazine 48 (1975), 87-92.

Department of Mathematics Tamkang University, Tamsui 25137, Taiwan, Republic of China. E-mail: 005490@math.tku.edu.tw

Department of Mathematics Tamkang University, Tamsui 25137, Taiwan, Republic of China. E-mail: liusolong@livemail.tw 